\title{
An Approach to Simulate the Manufacturing Process of Thermal Spray Coatings
}

\author{
Zhang Yanjun*, Zhang Shiqi, Yang Qiuyu and Hang Biyun \\ College of Mechanical Engineering, Yangzhou University, Yangzhou, 225127, China \\ ${ }^{*}$ Corresponding author
}

Keywords: Thermal spraying, Manufacturing process, Simulaiton

\begin{abstract}
Thermal spraying technology is one of the most efficient methods to surface modifying in the field of surface engineering, meanwhile, the application of spaying operation on the workpieces conducted by industrial robot enjoys increasingly popularity. Based on the analysis of thermal spraying components and parameters, the paper proposed an approach to simulate the manufacturing process of thermal spray coatings. In addition, the effectiveness and feasibility of this method has been demonstrated by a case study of the spraying process simulation on a certain blade surface.
\end{abstract}

\section{Introduction}

Accompanied with the rapid development of modern machinery industry, the material properties require constant improvement. Among them, thermal spraying on the surface of workpieces is a technology with higher comprehensive benefits. With the enhancement of control speed and precision of robot, thermal spraying operation conducted by industrial robot has been more and more widely used $[1,2]$.

Teaching programming was mainly used in the early stage of thermal spraying robot programming, the robot is set at manual-operated pattern and several points are set on the workpiece. The robot is operated to move to every point at the set parameters with the coordinates recorded immediately. Thus, the robot's motion trajectory is formed via the fitting of discrete points. This method can meet the demands of simple geometric body but isn't suitable generally for the complicated surface of workpiece due to the problem of low precision of motion trajectory and non-ideal effect of spraying.

Aiming at the problems in the process of thermal spraying using robot, this thesis will analyze the important parameters which can affect the quality of spraying, construct the virtual simulation circumstance of thermal spraying robot and spraying visualization, and propose the method of robot spatial trajectory toward the surface of workpiece, which make the realization of trajectory planning based on the off-line programming come true. In the end, the relatively ideal spraying trajectory can be obtained.

\section{The analysis of thermal spraying process}

Composition of thermal spraying system. Thermal spraying is the technology that use one certain heat source, such as arc, plasma arc, the combustion flame to have the power or filamentous mental or non- mental coating material heated to melt or semi-melt, sprayed to preprocessing surface of the substrate material at a certain speed with the help of the flame flow itself or imposed high speed airflow, which form the surface coating with a variety of functions combined with base material. Generally, spraying equipment and its auxiliary equipment are included in the thermal spraying system, showed in Figure 1. 


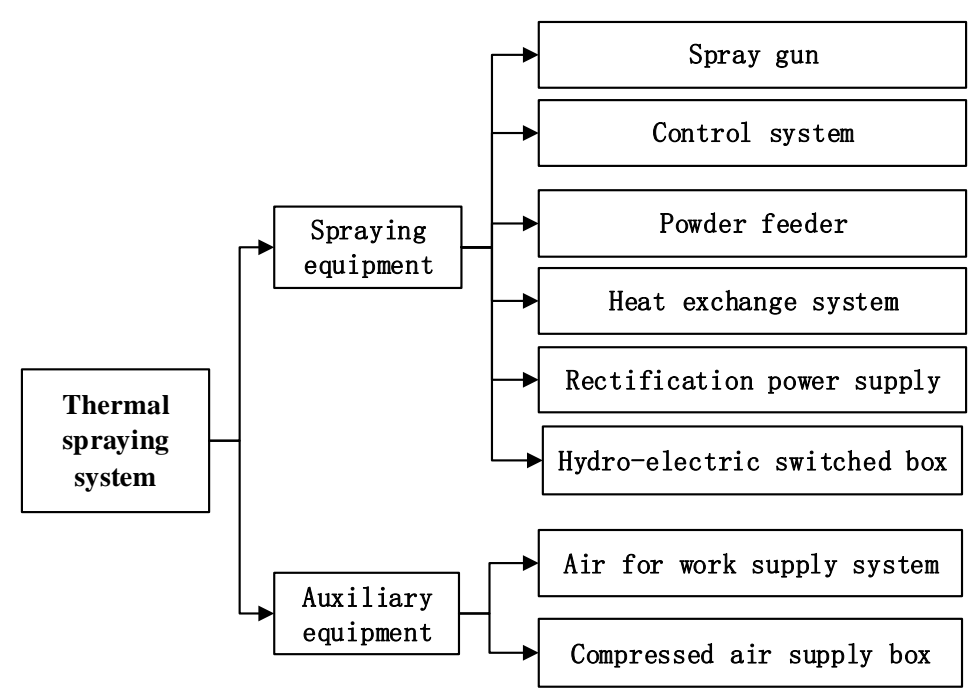

Figure 1 Composition of plasma spraying system

Thermal spraying parameters. The main parameters which effect thermal spraying cover the powder feeding rate, spraying distance, spraying angle and so on. According to the plasma spraying, nitrogen or argon gas are generally chose as the main gas, hydrogen as auxiliary gas; the size of feeding amount is an important parameter which can influence microstructures of coating and deposition efficiency, if the amount is too large, not only the efficiency of dust sedimentary will be reduced, but also increase the holes in the coating and the number of unmalting particle, which will result in lower quality of coating; if too small, besides the increase of cost, those bad effects will emerge such as overheating of the parts cracking of the coating and so on .The influence of spraying distance to the coating lies in the speed of the particle coming from the lance, which are different in the difference place. Too long or too short of the spraying distance will both have influence on the binding performance of coating, while spraying angle have an effect on the binding strength.

\section{Method of manufacturing process simulation}

The framework of trajectory planning. The trajectory planning of robot spraying includes the analysis of motion parameters, construction of simulation circumstance, modeling of workpiece and tools etc., the flow chart is shown in Figure 2.

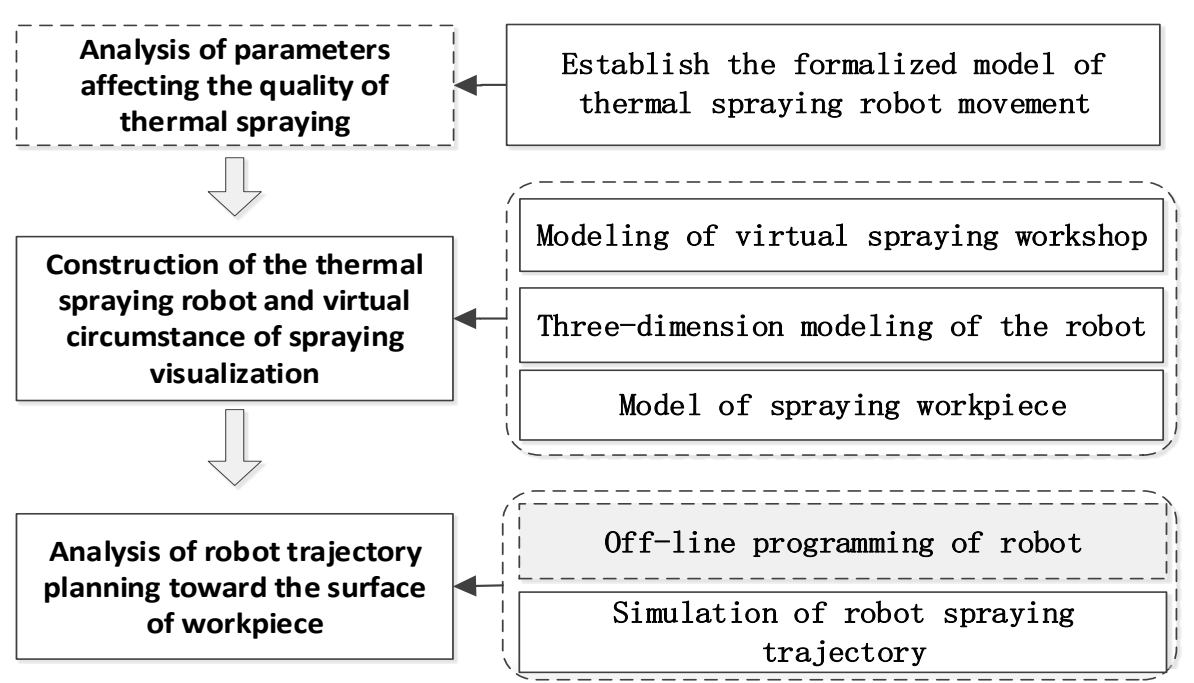

Figure 2 The flow chart of trajectory planning

Parameters influencing the quality of thermal spraying. On the basis of processing requirements, analysis and calculation on parameters are needed to make the uniform thickness of spraying layer, including the spraying angle, spraying distance, movement speed of lance. Thus, the 
model of kinematic parameters can be established. To get better coating structure, the direction of the spraying gun should be coincided with normal direction of the coating layer and the distance between the gun and the surface should be guaranteed at a certain fixed value; when determining the gun's movement speed, uniform velocity should be guaranteed at the line, curve, turning and so on; The intervals between the adjacent paths should be determined according to the spraying distance.

Thermal spraying simulation circumstance. Thermal spraying simulation circumstance is composed of painting workshop, three-dimension model of robot and worktable. Take the trajectory planning of $\mathrm{ABB}$ robot as an example, according to the workpiece and spraying requirements, based on comprehensive consideration, including working range, load on the robot, angles of each robot's axis required turning and other factors, the corresponding models of robot can be chose in the Robot Studio, besides, three-dimension model such as virtual spraying workshop and workbench also can be established in the software of CATIA or Robot studio, showed in Figure 3.

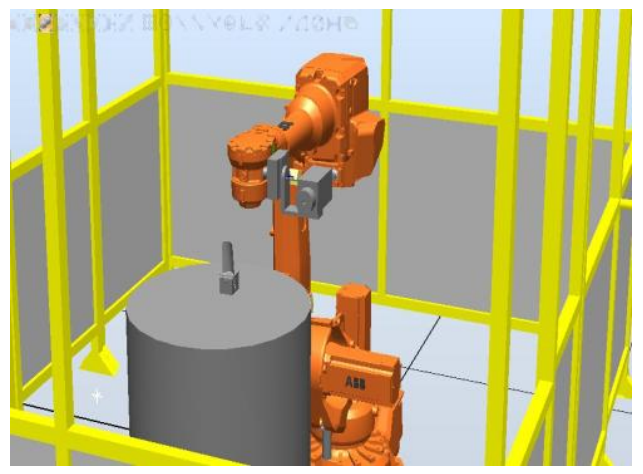

Figure 3 Simulation circumstance

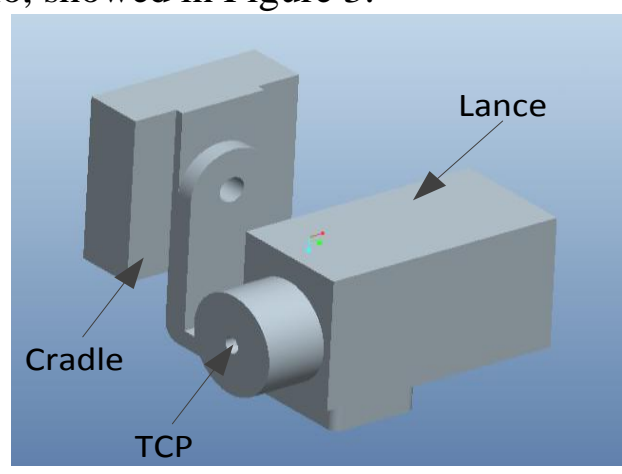

Figure 4 Model of lance cradle

According to the type of robot, comprehensively considered, those restricted conditions such as the lance and load on itself, the model of corresponding type of spraying gun can be created, which is showed in Figure 4. Mapping from the virtual environment to the actual painting work environment can be realized via the construction of simulation environment, basing on the installation position of workpiece in the actual painting work environment.

Trajectory simulation of thermal spraying. Trajectory simulation of thermal spraying contains three parts $[4,5]$ : establishment of workstation, building the spraying path and synchronization of the path, the flow chart is as followed in Figure 5.

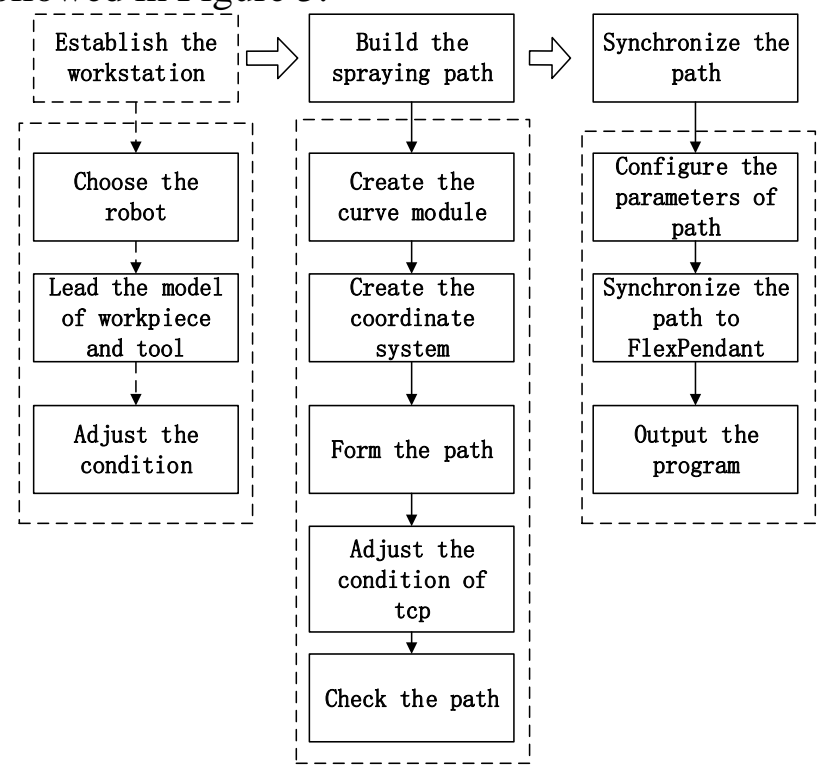

Figure 5 The flow chart of the path simulation

(1)Generate a new workstation in Robot studio and prepare for the building of spraying path.

a) Choose the corresponding type of the robot, log in the operation interface, adjust the initial position of robot's six axis in order to build the model of path. 
b) Lead the spraying tools and workpiece's three-dimension model into the workstation, set them reasonably and pay attention to the combining of workpiece and tools, so that space curve of high precision can be obtained when generate the spraying curve according to the three-dimension graphics.

(2) Build the spraying trajectory on the basis of workpiece model

a) Create the curve module: click the surface boundary in the 'modeling' module, choose the surface needed spraying to create three-dimension module. Then generate the motion trajectory according to the module and the workpiece's curve carried by CATIA.

b) Create the coordinate system: during the process of trajectory operation, usually, it is needed to create user coordinate system for later programming and modify the path. After that, pay attention to change the workpiece coordinate and tool coordinate, for that the different choice of these two coordinates will have an effect on the operation of later motion of actual trajectory.

c) Forming path: plan the path and form it. If the path is rather complex, dividing it into many segments and creating them.

d) Adjust the condition of TCP (tool center point): some of the trajectory are not ideal, to realize the goal of making spraying amount evenly distributed, it is necessary to inspect the orientation of target and adjust the condition of TCP so as to keep TCP vertical to the curve.

e) Check the trajectory: when the terminal of robot comes to one point, there are many different conditions of robot's each axis. So turning angle of each axis should be configured. The whole path can be obtained after multiple inspection.

f) After the parameters of each axis in target point being configured, the spraying path can be obtained and synchronized to VC (virtual control) in off-line module. Choose the tool coordinate, then the coordinate of workpiece and path program will also be synchronized to $\mathrm{VC}$, the control will produce the program of spraying path, meanwhile, the motion change law of robot's each axis in this process of movement can be obtained in the 'monitoring' module[4].

\section{A case study}

Take a certain blade for example, the method above can be demonstrated via the trajectory planning of thermal spraying with corresponding material on the blade surface. The model of blade is showed in the Figure 6.

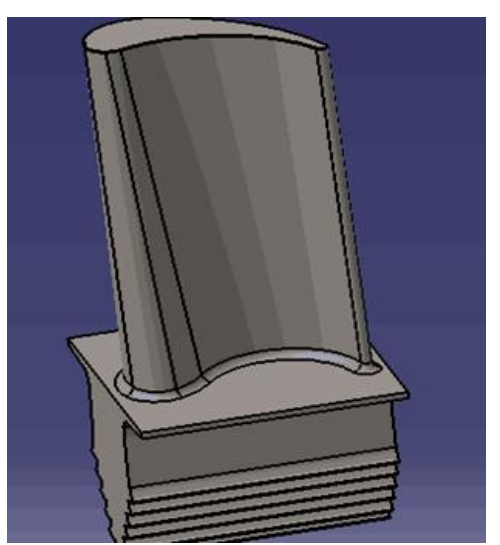

Figure 6 The blade model

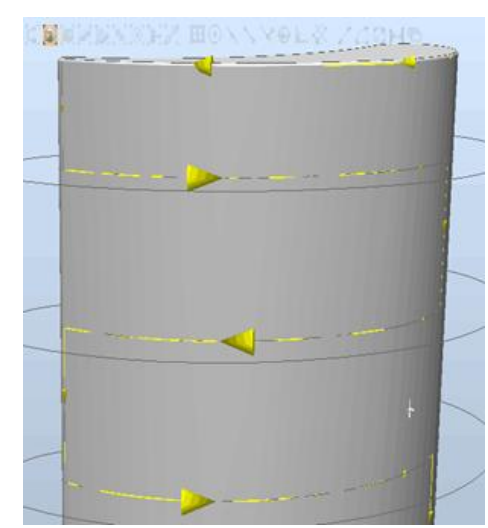

Figure 7 Simulation of spraying trajectory

Take the steps above to conduct simulation and the trajectory is showed in Figure 7 . The coordinates and attitude data (part) of target point along the path can be obtained by off-line programming, which are as follows:

MODULE Module1

CONST robtarget

Target_10:=[[41.001531786,12.587809333,77.788447569],[0.126205012,0.118867177,-0.67600 9998,-0.716207632],[-1,0,-1,0],[9E9,9E9,9E9,9E9,9E9,9E9]];

CONST robtarget 
Target_20:=[[30.144869714,12.955626752,77.938145789],[0.007635351,0.008809588,0.709080 34,0.705031321],[-1,0,-1,0],[9E9,9E9,9E9,9E9,9E9,9E9]];

CONST robtarget

Target_50:=[[6.666648987,38.262995869,77.941842772],[0.404118344,0.40738509,0.58143174 2,0.576769349],[0,1,-1,0],[9E9,9E9,9E9,9E9,9E9,9E9]];

The program (part) of blade's spraying path through trajectory simulation are as followed:

PROC Path_10()

MoveL Target_10,v1000,z100,pqt|WObj:=WobjFixture_1;

MoveC Target_20,Target_30,v1000,z100,pqt lWObj:=WobjFixture_1;

MoveC Target_40,Target_50,v1000,z100,pqt|WObj:=WobjFixture_1;

ENDPROC

ENDMODULE

\section{Conclusion}

In view of the problems existing in the process of thermal spraying, to get the ideal trajectory, this thesis analyzed the parameters affecting the quality of spraying, constructed the circumstance of spraying visualization simulation and proposed an approach to simulate the manufacturing process of thermal spray coatings. In addition, the effectiveness and feasibility of this method is demonstrated by the case study of thermal spraying simulation on one certain blade surface.

\section{References}

[1] Binshi Xu,.ShiCan liu,.surface engineering manual. Peking. chemical industry press.2009;

[2] Wei Jiang. JInPing Zhao. Min Gong. Thermal spraying technology and its development. China Coating.2006. 21(11): 51-52;

[3] L Pawlowski. The Science and Engineering of Thermal Spray Coatings. Wiley, 1995;

[4] Hui Ye. Industrial robot typical application case analysis. Peking. Mechanical industry Press.2013;

[5] ZiXing Cai. Robotics basis. Peking. Mechanical industry Press.2009 